\title{
Perfil dos acidentes de trabalho publicados em estudos brasileiros
}

\author{
Andrea Prochnow*, Tânia Solange Bosi de Souza Magnago**, \\ Juliana Petri Tavares ${ }^{* * *}$, Carmem Lúcia Colomé Beck*, Rosângela \\ Marion da Silva ${ }^{* \star *}$, Patrícia Bitencourt Toscani Greco ${ }^{* \star * * *}$
}

RESUMO: Objetivo: traçar o perfil das publicações brasileiras sobre acidentes de trabalho no período de 2004 a 2010. Método: revisão integrativa dos estudos publicados online, utilizando-se a palavrachave "acidente de trabalho". A busca foi realizada no banco de dados da SCIELO, BDENF e LILACS, entre julho e agosto de 2010. Através da similaridade de conteúdos, os estudos foram agrupados em temáticas. Resultados: Os resultados apontam para três temas centrais: características dos acidentes de trabalho, prevenção dos acidentes/tratamento adotado e subnotificação dos registros. Conclusão: 0 acidente típico foi o predominante, a enfermagem a profissão mais pesquisada, os instrumentos perfurocortantes e material biológico foram os principais agentes causadores de acidentes, a lavagem do local foi a conduta mais adotada após 0 acidente, a subnotificação foi elevada. A complexidade dos acidentes de trabalho suscita novas investigações acerca das condições de trabalho e sua associação com o risco da ocorrência de acidentes.

Descritores: Acidentes de trabalho; Saúde do trabalhador; Enfermagem; Condições de trabalho.

\section{Profile of occupational accidents published in brazilian studies}

ABSTRACT: Objective: The research aimed to set the profile of Brazilian publications on occupational accidents in the period from 2004 to 2010. Method: it was an integrative review of studies published online, using the keyword "occupational accident". The search was performed on the database of SciELO, BDENF, and LILACS between July and August 2010. By similarity of content, the studies were grouped into topics. Results: The results point to three central topics: characteristics of accidents, accident prevention/treatment adopted and the underreporting of records. Conclusion: The typical accident was predominant, the nursing profession was the most researched, the sharp instruments and biological material were the main agents causing accidents, washing the site was the most common measure taken after the accident, and underreporting was high. The complexity of labor accidents instigates new investigations on the conditions of work and their association with the risk of them happening.

Descriptors: Accidents, Occupational; Occupational Health; Nursing; Working Conditions. 


\section{Introdução}

Dentre as causas de adoecimento e afastamento do ambiente laboral está o acidente de trabalho que pode ser considerado o maior agravo à saúde dos trabalhadores e se constitui em um relevante problema de saúde pública. ${ }^{1}$

Por definição, acidente de trabalho é um evento súbito que ocorre durante o exercício da atividade laboral e que ocasiona dano à saúde do trabalhador (lesão corporal ou perturbação funcional) e pode ser responsável pela morte, perda ou redução da capacidade para 0 trabalho, permanente ou temporária. ${ }^{2}$

Considera-se também acidente de trabalho aquele que ocorre durante o deslocamento entre a residência e o local de trabalho e vice-versa, denominado acidente de trajeto. ${ }^{3}$

Todos os acidentes de trabalho, do menos grave ao fatal, são considerados agravos de notificação compulsória4 e a sua ocorrência deve ser comunicada à Previdência Social, por meio de abertura de Comunicação de Acidente de Trabalho (CAT). ${ }^{2}$

Falar em acidente de trabalho remete à saúde do trabalhador. Essa foi uma das justificativas para a escolha do assunto, uma vez que se vincula à linha de pesquisa Saúde do Trabalhador do Grupo de Pesquisa Trabalho, Saúde, Educação e Enfermagem do Departamento de Enfermagem da Universidade Federal de Santa Maria (UFSM) na qual os autores inserem-se. Além disso, tem-se a experiência profissional de uma das autoras como Técnica de Enfermagem do Pronto-Socorro de um hospital universitário onde atua há mais de oito anos. No exercer de seu trabalho, por diversas vezes prestou atendimento de pacientes que foram encaminhados ao serviço vítimas de acidentes de trabalho e, em outras, presenciou acidentes acometendo a equipe de enfermagem. Em alguns casos, no corre-corre do trabalho, os acidentes considerados "leves" ou "supostamente sem risco" acabam não sendo notificados pelo trabalhador ao setor competente da instituição.

A partir do exposto, as questões que nortearam a realização deste estudo foram: quais os acidentes de trabalho relatados em estudos brasileiros? Qual a população mais acometida? E, que medidas são adotadas para prevenir acidentes ou tratar trabalhadores que sofrem acidentes de trabalho? Para responder a estes questionamentos, objetivou-se traçar um perfil das publicações brasileiras acerca da temática acidente de trabalho, considerando o tipo de acidente, a categoria profissional acometida e o desfecho do acidente.

\section{Método}

Trata-se de uma revisão integrativa que buscou apresentar a revisão e análise crítica acerca dos artigos sobre acidente de trabalho, publicados online, no período de 2004 a 2010. Este método de pesquisa consiste na construção de uma vasta análise da literatura, contribuindo para discussões sobre métodos e resultados de pesquisas, além de reflexões sobre a realização de futuros estudos. ${ }^{5}$

O levantamento foi realizado na Biblioteca Virtual de Saúde (BVS), entre os meses de julho e agosto de 2010, no banco de dados Scientific Electronic Library Online (SCIELO), Base de Dados de Enfermagem (BDENF) e Literatura Latino-Americana e do Caribe em Ciências da Saúde (LILACS). Para o levantamento dos artigos, utilizou-se a palavra-chave "acidente de trabalho". 
Os critérios selecionados para a inclusão das publicações foram: artigos disponibilizados na íntegra e na forma online, sem restrições de acessibilidade; publicados no idioma português, no período compreendido entre os anos de 2004 a 2010, por considerar a Política Nacional de Segurança e Saúde do Trabalhador ${ }^{8}$, elaborada em 2004, um marco na segurança da saúde do trabalhador. Como critérios de exclusão elegeram-se: artigos de pesquisa bibliográfica e de reflexão, teses e dissertações. Para a inclusão ou exclusão dos artigos foram utilizados três Testes de Relevância. ${ }^{6}$ No Teste de Relevância 1, considerou-se 0 período de publicação e o idioma; no Teste de Relevância 2, levou-se em conta o título e/ou resumo para verificar a adequação ao tema de interesse (excluíram-se aquelas não disponíveis online, teses e dissertações, estudos de pesquisa bibliográfica e artigos repetidos); no Teste de Relevância 3, os estudos foram avaliados na íntegra.

As informações dos artigos selecionados foram sumarizadas em uma ficha de extração de dados denominada "Identificação e descrição do conteúdo do artigo"7 que assinala os seguintes itens: tema, título, autores, fonte da publicação, estudo número, finalidade/objetivo, coleta de dados/tipo de pesquisa, análise dos dados, resultados/discussão e conclusões/recomendações.

\section{Resultados e discussões}

A busca possibilitou a análise de 53 artigos (Quadro 1) conforme os critérios de inclusão, descritos anteriormente. Considerando-se a similaridade de conteúdos encontrada nos estudos, os resultados serão apresentados e discutidos com base em três temas centrais, quais sejam: características dos acidentes de trabalho, prevenção dos acidentes/tratamento adotado e subnotificação dos registros.

\section{Características dos acidentes de trabalho}

Do tema características dos acidentes de trabalho emergiram três categorias: tipos de acidente de trabalho, profissão do trabalhador acidentado e causas do acidente de trabalho.

Sobre os tipos de acidente de trabalho, $100 \%$ dos artigos selecionados abordaram os acidentes de trabalho típico, ou seja, 53 artigos. Em oito desses estudos (15,09\%), abordouse simultaneamente 0 acidente de trajeto.

A maioria dos acidentes sendo constituída por acidente de trabalho típico denota, primeiramente, a necessidade das instituições investirem na prevenção desse tipo de acidente pelo elevado custo (financeiro e humano), já que o número de dias de trabalho perdidos é responsável por aumentar o custo da mão de obra no Brasil, o que encarece a produção e diminuir a competitividade do país em relação ao mercado externo8; por segundo, levanta a hipótese de que os acidentes de trajeto possam estar subnotificados ou as pesquisas dão mais ênfase em investigações que envolvem os acidentes típicos.

$\mathrm{Na}$ categoria profissão do trabalhador acidentado, constatou-se que o maior percentual dos estudos $(35,85 \%, \mathrm{~N}=19)$ foi realizado com trabalhadores da equipe de enfermagem. Essa evidência demonstra que, apesar das instituições de saúde serem entidades que visam a assistência, o tratamento e a cura dos pacientes, elas também podem, de acordo com as condições de trabalho (dentre outros fatores), favorecer o adoecimento dos trabalhadores. ${ }^{9}$

Saúde (Santa Maria), v.37, n.1, p. 77-90, 2011. Prochnow, A. et al. 
Entre os estudos realizados com trabalhadores de enfermagem, a categoria que mais sofreu acidente de trabalho foi a de auxiliar de enfermagem, compreendendo percentuais entre $48 \%$ e $81 \%$ dos trabalhadores acidentados estudados. Em dois estudos, houve a predominância de acidentes com técnicos de enfermagem (64\% e 71,6\%), e em um estudo, os auxiliares e técnicos foram agrupados, evidenciando-os como os mais expostos a acidentes de trabalho no âmbito hospitalar (43,4\%).

Talvez esses dados possam ser explicados pelo fato de que os auxiliares e técnicos são os trabalhadores de enfermagem que desenvolvem com maior frequência as atividades ligadas à assistência direta ao paciente como, por exemplo, higienização, preparo e administração de medicamentos, curativos e atividades que envolvam manuseio de instrumentos cirúrgicos; tais atividades tornam o trabalhador mais suscetível a ocorrência de acidentes. ${ }^{10}$

As demais profissões foram: trabalhadores de saúde, incluindo auxiliar técnico de laboratório, farmacêutico/bioquímico, médico, técnico de análises clínicas, técnico em laboratório, técnico em higiene dental, auxiliar de consultório dentário, biomédico, fisioterapeuta, fonoaudiólogo, técnico em hemoterapia, atendente de enfermagem, técnico de eletrocardiograma, terapeuta ocupacional, serviços de apoio (cozinha, lavanderia, limpeza e manutenção) $(18,86 \%, \mathrm{~N}=10)$, trabalhadores em geral que procuraram atendimento após 0 acidente $(7,55 \%, \mathrm{~N}=4)$, odontólogos $(7,55 \%, \mathrm{~N}=4)$, profissionais de hospital, incluindo os serviços gerais $(3,77 \%, \mathrm{~N}=2)$, equipe multiprofissional de atendimento pré-hospitalar $(3,77 \%$, $\mathrm{N}=2$ ) e diversas categorias profissionais $(22,64 \%, \mathrm{n}=12)$, sendo um estudo para cada categoria, como por exemplo, fabricantes de pranchas de surf, operadores de poda de árvore, pescadores, trabalhadores da construção civil.

Sobre as causas dos acidentes de trabalho, evidenciaram-se: agressividade (por parte da vítima, familiar e usuário contra o trabalhador), ausência de Equipamento de Proteção Individual (EPI), cansaço físico e mental, choque térmico, colisão automobilística, contato com répteis venenosos, contusões e entorses, fluido ou material biológico, instrumentos perfurocortantes, lesões por animais, medicamentos, necessidade de maior agilidade de realização das atividades de rotina, pouca experiência profissional, quedas, queimaduras, radiação, ruído e substâncias químicas.

Considerando-se que em alguns estudos foram apontados mais de um agente causador, os instrumentos perfurocortantes e os fluidos/materiais biológicos foram os que apresentaram maior percentual, com $50 \%$ e 48,1\%, respectivamente. Este achado é importante e necessita de maior atenção tanto por parte dos gestores quanto dos trabalhadores de instituições de saúde devido à elevada frequência com que ainda aparecem nas estatísticas, ao grau de estresse do trabalhador pós-acidente e aos custos gerados. ${ }^{11}$ Custos estes, tanto para empresa quanto para o trabalhador, que muita vezes fica incapacitado de trabalhar.

Evidenciou-se, nesta revisão, que independente do local de trabalho, os trabalhadores podem estar expostos a diferentes fatores de riscos associados a um acidente de trabalho. A adoção de medidas de prevenção dos acidentes é fator crucial. Para isso, o entendimento dos processos de trabalho de cada profissão, a oferta de condição de trabalho adequada (recursos humanos, material e equipamento em quantidade e qualidade suficiente), a capacitação dos trabalhadores, o uso de EPI, atenção durante o exercício do trabalho, cuidado no manuseio, transporte e descarte de materiais e equipamentos, esquema vacinal completo do trabalhador, entre outras são de fundamental importância para reduzir o risco de acidentes. 
Importante destacar que alguns estudos assinalam as situações de estresse físico e/ou psíquico como causadores de risco no trabalho, dentre eles os de acidentes. Como situações estressantes foram citadas: o controle rígido de produtividade, imposição de ritmos excessivos, trabalho em turno e noturno, jornadas de trabalho prolongadas, monotonia e repetitividade. ${ }^{12}$, convívio com situações de dor e de morte dos pacientes. ${ }^{13}$

No que tange ao trabalhador enfermeiro, pode-se dizer que esse convive com situações associadas ao estresse ocupacional, como inovações organizacionais e tecnológicas, os crescentes conflitos relacionados às questões pessoais, sociais e institucionais no âmbito hospitalar, o que exige desses trabalhadores constante adaptação, maior consciência, além de grande habilidade para enfrentar essas evoluções e administrar as demandas do trabalho. ${ }^{14}$

Estudo ${ }^{15}$ que teve como objetivo identificar a frequência e as principais causas de acidentes ocupacionais com material biológico entre profissionais que atuam em laboratórios de análises clínicas obteve que 4,4\% dos entrevistados apontaram o estresse como motivos que determinaram a ocorrência das exposições ocupacionais envolvendo material biológico.

Em outro estudo ${ }^{16}$ que identificou os acidentes do trabalho ocorridos com trabalhadores de enfermagem de uma unidade de terapia intensiva, mostrou que 13\% dos trabalhadores apontaram a pressa decorrente do plantão ou o estresse como o motivo que ocasionou 0 acidente.

Já em outro estudo ${ }^{17}$, o estresse é assinalado como consequência do acidente sofrido, ou seja, a ocorrência do acidente aliado ao desconhecimento da sorologia do paciente podem constituir em motivo de estresse para o trabalhador acidentado, o que pode repercutir em problemas na sua saúde mental e afetar as relações familiares e laborais.

Assim, percebe-se que o estresse pode estar relacionado ao acidente de trabalho tanto como causa quanto como em consequência deste.

\section{Prevenção dos acidentes/tratamento adotado}

No tema prevenção dos acidentes e tratamento adotado emergiram três categorias: uso de Equipamentos de Proteção Individual, esquema de vacinação do trabalhador e condutas adotadas após 0 acidente.

A categoria uso de Equipamentos de Proteção Individual apontou para a necessidade da utilização de máscaras, avental, óculos de proteção e luvas, uma vez que o uso de EPI, aliados ao emprego de práticas seguras, reduzem significativamente o risco de acidente ocupacional. ${ }^{18}$

Essa necessidade é apontada em $37,74 \%(\mathrm{~N}=20)$ dos estudos, o que sugere a importância desse cuidado para a redução/eliminação dos acidentes, o que pode contribuir para a manutenção da saúde do trabalhador. Identificou-se em estudo ${ }^{19}$ que os profissionais, em sua maioria (92,1\%), conhecem os EPI e sabem da sua importância; contudo, há evidências do uso inadequado destes como um dos motivos que determina a ocorrência do acidente de trabalho. ${ }^{15}$

Importante destacar um estudo ${ }^{9}$ que buscou verificar a ocorrência de soroconversão pelos vírus da hepatite $B(\mathrm{HBV})$, hepatite $\mathrm{C}(\mathrm{HCV})$ e HIV, causador da Síndrome da Imunodeficiência Humana Adquirida (AIDS) após o acidente. Nele, obteve-se que, dos 87 Saúde (Santa Maria), v.37, n.1, p. 77-90, 2011. acidentes avaliados, oito ocorreram com pacientes portadores dessas patologias. Em dois 
casos, houve exposição de mucosa ocular do profissional, sendo que um paciente era portador de HCV e outro de HIV, e em nenhum dos dois acidentes os profissionais envolvidos utilizavam proteção ocular.

Assim, o profissional que apresenta um comportamento de risco, ou seja, que não faz uso de EPI está exposto aos acidentes de trabalho e possíveis acometimentos em decorrências desses. Embora o risco de transmissão ocupacional do HIV seja de 0,3\% para a exposição percutânea e $0,09 \%$ para mucocutânea ${ }^{20}$ a possibilidade de contaminação existe e não deve ser banalizada pelos trabalhadores.

Com isso, a constatação evidenciada nos estudos revisados sobre a baixa adesão às medidas de precaução por parte dos trabalhadores, traduz-se na importância do desenvolvimento de novas estratégias que busquem aumentar a adesão aos EPI, como forma de proteção e segurança no desenvolvimento do trabalho.

A segunda categoria, esquema de vacinação do trabalhador, foi composta por 12 estudos $(22,64 \%)$ que verificaram a situação vacinal dos trabalhadores. Destes, 10 estudos investigaram o esquema vacinal para hepatite $B$ e dois avaliaram o esquema de vacinação para o tétano. Como resultado, obteve-se que entre 30,6\% e 100\% dos sujeitos apresentavam esquema completo para hepatite $B$.

A amplitude dos percentuais de trabalhadores com esquema completo para hepatite $B$ é grande $(70 \%)$, o que evidencia a existência de grande parte dos trabalhadores que continuam expostos ao risco de contaminação ocupacional. Cabe salientar que a imunização é uma das principais medidas de prevenção para a hepatite $\mathrm{B}^{17}$, e tem uma eficácia de 90 a 95\%. ${ }^{15}$

Após a realização do esquema completo de imunização contra hepatite $B$, se faz necessária a realização do teste anti-HBs para verificação da resposta vacinal. Esse teste é de grande valia, pois estudo ${ }^{21}$ identificou que a maioria dos acidentados relatou vacinação prévia contra a hepatite B $(91,3 \%)$, entretanto, $73 \%$ apresentaram resultado reagente para 0 anticorpo anti-HBs. Muitos profissionais, apesar da vacinação, não estão protegidos contra a infecção pelo HBV, provavelmente por não terem completado o esquema vacinal ou por não apresentarem resposta vacinal.

Ressalta-se que todo o trabalhador dos serviços de saúde deve receber gratuitamente, como medida preventiva, a imunização ativa contra tétano, difteria, HBV e os estabelecidos no Programa de Controle Médico de Saúde Ocupacional. ${ }^{18}$

A categoria condutas adotadas após 0 acidente foi investigada e apresentada em 20 estudos $(37,74 \%)$, identificando as atitudes que os trabalhadores adotaram após os acidentes, incluindo a adesão ao protocolo pós-exposição e a quimioprofilaxia.

A conduta predominante realizada pelos trabalhadores após um acidente de trabalho foi a lavagem do local atingido. Tal conduta condiz com o preconizado pelo Ministério da Saúde (MS) que indica em caso de exposição percutânea, a lavagem exaustiva com água e sabão e, em caso de exposição em mucosas, realizar lavagem com água ou solução salina. ${ }^{20}$

Outras condutas evidenciadas foram: aplicação de álcool 70\%, antisséptico, hipoclorito ou solução salina fisiológica, investigação do paciente sobre HIV/hepatites, assepsia com e sem a comunicação do Controle de Infecção do Hospital $(\mathrm{CCIH})$, busca de atendimento médico, espremer o local atingido, nenhuma conduta foi adotada, pois paciente parecia sadio, notificação do acidente mediante CAT, realização de curativo, realização de exames, utilização de antiretrovirais, utilização de algum tipo de prática popular como aguardente, partes de peixes e urina e vacinação anti-tetânica. 
Percebe-se que muitas condutas são adotadas após o episódio de um acidente, sendo que algumas delas não são recomendadas pelo MS e outras são até contra-indicadas, como é o caso da lavagem do local com hipoclorito ou outras soluções irritantes. ${ }^{20} \mathrm{~A}$ expressão do local atingido como forma de facilitar o sangramento não é uma conduta justificada em nenhum estudo como forma de facilitar o sangramento espontâneo. ${ }^{22}$

Após a ocorrência de um acidente, condutas podem prevenir infecções e devem ser adotadas, incluindo a avaliação imediata do acidente, quimioprofilaxia quando necessário, aconselhamento do trabalhador e do paciente, bem como 0 acompanhamento periódico do acidentado. ${ }^{23}$ Contudo, estudo ${ }^{24}$ realizado com a equipe multiprofissional de Atendimento PréHospitalar concluiu que em $55,1 \%$ dos acidentes não foi tomada nenhuma conduta imediatamente após o acidente, e que em $61,2 \%$ dos acidentados 0 acompanhamento sorológico não foi realizado.

Ressalta-se que nenhuma medida pós-exposição é totalmente eficaz e que, portanto, medidas de proteção individual e coletiva no desenvolvimento das atividades são fundamentais e a prevenção ainda é a melhor medida para evitar a transmissão ocupacional. ${ }^{25}$

E, embora exista um manual de condutas recomendados pelo MS em caso de exposição ocupacional a material biológico, constatou-se que os trabalhadores podem adotar outros tipos de condutas. Dessa maneira, pode-se inferir que há um desconhecimento desta normatização por parte dos trabalhadores, o que pode repercutir não somente em prejuízo para a profilaxia pós-exposição ao acidente de trabalho, mas como em dificuldades à adesão de medidas relacionadas à prevenção.

\section{Subnotificação dos registros}

No tema subnotificação dos registros, 14 estudos (26,42\%) abordaram a importância da comunicação do acidente de trabalho e, principalmente, as consequências que a subnotificação acarreta. Ela foi verificada em percentuais compreendidos entre $48,9 \%$ e $84,6 \%$. Ressalta-se que $19,2 \%$ dos estudos, considerando os resultados obtidos, apenas sugerem a existência de subnotificação, não explicitando os índices.

Importante destacar um estudo ${ }^{10}$ que observou diversidade na notificação dos acidentes, falhas no seguimento pós-acidente, falta de exames anexados à ficha ou não cumprimento em tempo hábil e conforme protocolo institucional e falta de padronização em algumas condutas quanto à solicitação de exames.

Como justificativa para a não notificação do acidente de trabalho, foram citados nos estudos: burocracia, contato com sangue ou fluido corpóreo ou de excreta em pele íntegra, desconhecimento do protocolo de rotina ou da necessidade de notificação, desinteresse, displicência, falta de tempo, funcionamento em horário comercial da Comissão de Controle de Infecção Hospitalar, irrelevância do acidente, medo do afastamento do trabalho com consequentes perdas salariais e benefícios, plantão agitado com intercorrências, médico plantonista que descartou a necessidade, sorologia negativa do paciente e sobrecarga de trabalho.

0 desconhecimento da necessidade de registro, além das demais justificativas apontadas, revela a necessidade de intervenções institucionais para o aumento desse registro, buscando-se que o trabalhador adquira uma maior compreensão sobre autocuidado e reflexão quanto a sua prática profissional e 0 aspecto legal envolvido no acidente de trabalho. ${ }^{26}$

Saúde (Santa Maria), v.37, n.1, p. 77-90, 2011.

Prochnow, A. et al. 


\section{Considerações Finais}

Após a realização deste estudo, pode-se concluir que a categoria profissional mais pesquisada no que tange aos Acidentes de Trabalho foi a enfermagem, que os agentes causadores desses acidentes foram os instrumentos perfurocortantes e material biológico, que a lavagem do local foi a conduta mais adotada após o acidente, que o tipo de acidente prevalente foi o típico e que a subnotificação é elevada.

Os estudos apontaram para a necessidade de utilização de EPI e para a importância dos trabalhadores manterem o esquema de vacinação completo. Nesse sentido, além do dever da instituição em oferecer os EPI é necessário a capacitação da equipe e a vigilância constante sobre o uso dos mesmos. No entanto, a responsabilidade não é somente da instituição, pois cabe ao trabalhador ter consciência sobre a importância da utilização dos EPI no desempenho de suas atividades.

Também é fundamental que além do esquema vacinal, seja efetuado o teste para verificação da resposta vacinal. Ressalta-se a importância de que após a exposição ocupacional, condutas recomendadas pelo Ministério da Saúde devam ser adotadas pelos trabalhadores.

A subnotificação foi outro aspecto avaliado nos estudos, assim como as justificativas apontadas pelos trabalhadores para não notificar o acidente, representando mais uma vez, a falta de conscientização por parte dos trabalhadores. 0 desconhecimento da necessidade de notificação deve ser revisto com especial atenção já que aponta para uma falha nas ações educativas. $O$ enfermeiro, como trabalhador comprometido com sua saúde e das pessoas, tem papel fundamental nessas ações, pois a prevenção dos acidentes de trabalho refletirá na redução dos percentuais dos acidentes e da subnotificação destes.

Como limitação deste estudo, aponta-se o fato de que o maior número de publicações direcionou-se ao ambiente hospitalar e à enfermagem, o que fez com que as discussões dos resultados fossem apontadas, em sua maioria, para esta profissão. Por outro lado, à medida que estabelece um perfil dos acidentes de trabalho, traz contribuições à enfermagem no sentido de que essas informações podem ser utilizadas como ferramentas para auxiliar na organização e gerenciamento dos serviços de enfermagem, auxiliando na elaboração de estratégias para a resolução desse problema, com base na realidade identificada.

Por fim, a complexidade dos acidentes de trabalho suscita novas investigações acerca das condições de trabalho e sua associação com tais acidentes em diversas populações.

\section{Referências}

1. Dias A, Cordeiro R, Gonçalves CGO. Exposição ocupacional ao ruído e acidentes do trabalho. Cad. Saúde Pública. 2006; 22(10): 2125-30.

2. Brasil. Ministério da Saúde. Secretaria de Atenção à Saúde. Departamento de Ações Programáticas Estratégicas. Notificação de acidentes do trabalho fatais, graves e com crianças e adolescentes. [online] 2006 [acesso em 2010 Out 06]. Disponível em: <http://bvsms.saude.gov.br/bvs/publicacoes/06_0442_M.pdf>.

Saúde (Santa Maria), v.37, n.1, p. 77-90,

2011. 3. Giomo DB, Freitas FCT, Alves LA, Robazzi MLCC. Acidentes de trabalho, riscos ocupacionais e estudos brasileitos absenteísmo entre trabalhadores de enfermagem hospitalar. Rev. enferm. UERJ. 2009; 17(1): 24-9. 
específica, no Sistema Único de Saúde - SUS. [online] 2004 [acesso 2011 Mar 10]. Disponível em: ///http://dt2001.saude.gov.br/sas/PORTARIAS/Port2004/GM/GM-777.htm>

5. Mendes KDS, Silveira RCCP, Galvão CM. Revisão integrativa: método de pesquisa para a incorporação de evidência na saúde e na enfermagem. Texto Contexto Enferm. 2008; 17(4):758-64.

6. Pereira AL, Bachion MM. Atualidades em revisão sistemática de literatura, critérios de força e grau de recomendação de evidência. Rev Gaúcha Enferm. 2006; 27(4): 491-8.

7. Fonseca RMP. Revisão integrativa da pesquisa em enfermagem em centro cirúrgico no Brasil: trinta anos após o SAEP. São Paulo: [s.n.]. 135f. Dissertação (Mestrado) - Escola de Enfermagem da Universidade de São Paulo, 2008.

8. Brasil. Ministério da Previdência Social. Política Nacional de Segurança e Saúde do Trabalhador. Brasília: Ministério da Saúde. [online] 2004 [acesso em 2010 out 09] Disponível em: $<$ <ttp://www.previdenciasocial.gov.br/arquivos/office/3081014-105206-701.pdf>

9. Magagnini MAM, Ayres JA. Acidentes com material biológico: a realidade de uma instituição hospitalar do interior paulista. REME - Rev. Min. Enferm. 2009; 13(1): 123-31.

10. Ribeiro PC, Ribeiro ACC, Lima Júnior FPB. Perfil dos acidentes de trabalho em um hospital de Teresina, PI. Cogitare Enferm. 2010; 15(1): 110-6.

11. Dalarosa MG, Lautert L. Acidente com material biológico no trabalhador de enfermagem em um hospital de ensino: estudo caso-controle. Rev Gaúcha Enferm. 2009; 30(1): 19-26.

12. Soerensen AA, Moriya TM, Soerensen R, Robazzi MLCC. Atendimento pré-hospitalar móvel: fatores de riscos ocupacionais. Rev. enferm. UERJ, Rio de Janeiro, 2008; 16(2):187-92.

13. Sarquis LMM, Felli VEA. Os sentimentos vivenciados após exposição ocupacional entre trabalhadores de saúde: fulcro para repensar o trabalho em instituições de saúde. Rev Bras Enferm. 2009; 62(5): 701-4.

14. Guido LA, Umann J, Stekel LMC, Linch GFC, Silva RM, Lopes LFD. Estresse, coping e estado de saúde de enfermeiros de clínica médica de um hospital universitário. Cienc Cuid Saude. 2009; 8(4):615-21.

15. Prado-Palos MA, Canini SRMS, Gir E, Melo LL, Mata DH, Santana RMT et al. Acidentes com Material Biológico Ocorridos com Profissionais de Laboratórios de Análises Clínicas. DST j. bras. doenças sex. transm. 2006; 18(4): 231-4.

16. Nishide VM, Benatti MCC, Alexandre NMC. Ocorrência de acidente de trabalho em uma unidade de terapia intensiva. Rev Latino-am Enfermagem 2004; 12(2):204-11.

17. Murofuse NT, Marziale MHP, Gemelli LMG. Acidente com material biológico em hospital universitário do oeste do Paraná. Rev Gaúcha Enferm, Porto Alegre (RS) 2005; 26(2):168-79.

18. Simão SAF, Souza V, Borges RAA, Soares CRG, Cortez EA. Fatores associados aos acidentes biológicos entre profissionais de enfermagem. Cogitare Enferm. 2010; 15(1): 87-91.

19. Pereira AKM, Silva AR, Rocha CF, Cordeiro IS, Lopes CM. Acidentes de trabalho com material perfurocortante em profissionais da equipe de enfermagem da rede hospitalar pública de Rio Branco - Acre Brasil. Online braz. j. nurs. (Online), 2004; 3(3).

20. Brasil. Ministério da Saúde. Manual de condutas em exposição ocupacional a material biológico. [online] 2003 [acesso em 2011 Mar 17]. Disponível em: http://www.opas.org.br/gentequefazsaude/bvsde/bvsacd/../condutas.pdf

21. Silva JA, Paula VS, Almeida AJ, Villar LM. Acidentes biológicos entre profissionais de saúde. Esc Anna Nery Rev Enferm, 2009; 13(3): 508-16.

22. Brasil. Ministério da Saúde. Recomendações para atendimento e acompanhamento de exposição ocupacional a material biológico: HIV e hepatites B e C. [online] 2004. [acesso em 2011 Mar 17]. Disponível em: http:// www.aids.gov.br/../recomendacoes-para-atendimento-e-acompanhamento-de -exposicaoocupacional-material-biologi

Saúde (Santa Maria), v.37, n.1, p. 77-90, 2011. Prochnow, A. et al. 
23. Martins AMEBL, Pereira RD, Ferreira RC. Adesão a protocolo pós-exposição ocupacional de acidentes entre cirurgiões dentistas. Revista Saúde Pública, 2010; 44(3): 529-40.

24. Oliveira AC, Lopes ACS, Paiva MHRS. Acidentes ocupacionais por exposição a material biológico entre a equipe multiprofissional do atendimento pré-hospitalar. Rev. Esc. Enferm. USP, 2009; 43(3): 677-83.

25. Garcia LP, Blank VLG. Condutas pós-exposição ocupacional a material biológico na odontologia. Rev. Saúde Pública. 2008; 42(2): 279-86.

26. Oliveira AC, Gonçalves JA. Acidente ocupacional por material perfurocortante entre profissionais de saúde de um Centro Cirúrgico. Rev Esc Enferm USP 2010; 44(2): 482-7.

\section{Andrea prochnow}

Endereço para correspondência- Rua Travessa Farrapos, 19. Bairro Medianeira. Santa Maria-RS. CEP $97015-240$

E-mail: andrea.prochnow@yahoo.com.br

Recebido em 18 de abril de 2011.

Aceito em 12 de setembro de 2011. 
ANEXO

Quadro 1: Relação dos estudos encontrados no período de janeiro 2004 a julho de 2010.

\section{REFERÊNCIAS DOS ARTIGOS INCLUÍDOS NO ESTUDO}

1. Almeida CAF, Benatti MCC. Exposições ocupacionais por fluidos corpóreos entre trabalhadores da saúde e sua adesão à quimioprofilaxia. Rev. Esc. Enferm. USP. 2007; 41(1): 120-6.

2. Almeida CB, Pagliuca LMF, Leite ALAS. Acidentes de trabalho envolvendo os olhos: avaliação de riscos ocupacionais com trabalhadores de enfermagem. Rev.Latinoam. enferm. 2005; 13(5): 708-16.

3. Almeida IS, Maia HF. Perfil clínico epidemiológico de acidentados no pólo petroquímico de Camaçari, Bahia. Rev. Baiana de Saúde Pública. 2009; 33(2): 106-21.

4. Alves CA, Osorio C. Análise coletiva de acidentes de trabalho: dispositivo de intervenção e formação no trabalho. Cadernos de Psicologia Social do Trabalho. 2005; 8: 87-98.

5. Bakke HA, Araújo NMC. Acidentes de trabalho com profissionais de saúde de um hospital universitário. Produção. 2010; 20(4): 669-76.

6. Balsamo AC, Felli VEA. Estudo sobre os acidentes de trabalho com exposição aos líquidos corporais humanos em trabalhadores da saúde de um hospital universitário. Rev. Latinoam. Enferm. 2006; 14(3): 34653.

7. Barboza DB, Soler ZASG, Ciorlia LAS. Acidentes de trabalho com pérfuro-cortante envolvendo a equipe de enfermagem de um hospital de ensino. Arq. Ciênc. Saúde. 2004; 11(2): 93-9.

8. Bonini AM, Zeviani CP, Facchin LT, Gir E, Canini SRMS. Exposição ocupacional dos profissionais de enfermagem de uma unidade de terapia intensiva a material biológico. Rev. eletrônica enferm. 2009; 11(3): 658 .

9. Bucasio E, Vieira I, Berger W, Martins D, Souza C, Maia D et al. Transtorno de estresse pós-traumático como acidente de trabalho em um bancário: relato de um caso. Rev Psiquiatr. 2005; 27(1):86-9.

10. Caixeta RB, Barbosa-Branco A. Acidente de trabalho, com material biológico, em profissionais de saúde de hospitais públicos do Distrito Federal, Brasil, 2002/2003. Cad. Saúde Pública. 2005; 21(3):737-46.

11. Castro MR, Farias SNP. Repercussões do acidente com perfurocortantes para a enfermagem: uma construção a partir do grupo focal. Esc. Anna Nery Rev. Enferm. 2009; 13(3): 523-9.

12. Dalarosa MG, Lautert L. Acidente com material biológico no trabalhador de enfermagem em um hospital de ensino: estudo caso-controle. Rev Gaúcha Enferm. 2009; 30(1): 19-26.

13. Damasceno AP, Pereira MS, Souza ACS, Tiplle AFV, Prado MA. Acidentes ocupacionais com material biológico: a percepção do profissional acidentado. Rev Bras Enferm. 2006; 59(1): $72-7$.

14. Dias A, Cordeiro R, Gonçalvez CGO. Exposição ocupacional ao ruído e acidentes de trabalho. Cad. saúde pública. 2006; 22(10): 2125-30.

15. Dias FLA, Pinheiro PNC, Barroso MGT. Perfil dos profissionais de enfermagem que se acidentam com materiais perfurocortantes no seu ambiente de trabalho. Rev. RENE. 2006; 7(3): 9-14.

Saúde (Santa Maria), v.37, n.1, p. 77-90, 2011.

Prochnow, A. et al. 
16. Fiedler NC, Sone EH, Vale AT, Juvêncio JF, Minette LJ. Avaliação dos riscos de acidentes em atividades de poda de árvores na arbonização urbana do Distrito Federal. R. Árvore. 2006; 30(2): 223-33.

17. Garcia LP, Blank VLG. Condutas pós-exposição ocupacional a material biológico na odontologia. Rev. Saúde Pública, 2008; 42(2): 279-86.

18. Garrone Neto D, Cordeiro RC; Haddad Júnior, V. Acidentes do trabalho em pescadores artesanais da região do Médio Rio Araguaia, Tocantins, Brasil. Cad. Saúde Pública, 2005; 21(3): 795-803.

19. Gerente VM, Melo GB, Regatieri CVS, Alvarenga LS, Martins EN. Trauma ocupacional por corpo estranho corneano superficial. Arq Bras Oftalmol. 2008; 71(2):149-52.

20. Giomo DB, Freitas FCT, Alves LA, Robazzi MLCC. Acidentes de trabalho, riscos ocupacionais e absenteísmo entre trabalhadores de enfermagem hospitalar. Rev. enferm. UERJ. 2009, 17(1): 24-9.

21. Gomes AC, Agy LL, Malaguti SE, Canini SRMS, Cruz EDA, Gir E. Acidentes ocupacionais com material biológico e equipe de enfermagem de um hospital-escola. Rev. Enferm. UERJ. 2009; 17(2): 220-3.

22. Hennington EA, Cordeiro R, Moreira Filho DC. Trabalho, violência e morte em Campinas, São Paulo, Brasil. Cad. Saúde Pública. 2004; 20(2): 610-17.

23. Juvêncio JF, Duarte MFS. 0 trabalho informal de fabricantes de pranchas de surfe: uma análise das condições de saúde e trabalho. Rev. Bras. Cineantropom. Desempenho Hum. 2006; 8(3): 79-84.

24. Kirchhof ALC, Capellari C. Descrição das comunicações de acidentes de trabalho registradas no Instituto Nacional de Seguridade Social de Santa Maria, RS, no ano de 2000. Rev. Gaúch. Enferm. 2004; 25(2):194-201.

25. Lima FA, Pinheiro PNC, Vieira NFS. Acidentes com material perfurocortante: conhecendo os sentimentos e as emoções dos profissionais de enfermagem. Esc Anna Nery Rev. Enferm. 2007; 11(2): 205 $-11$.

26. Magagnini MAM, Ayres JA. Acidentes com material biológico: a realidade de uma instituição hospitalar do interior paulista. REME - Rev. Min. Enferm. 2009; 13(1): 123-30.

27. Martins AMEBL, Pereira RD, Ferreira RC. Adesão a protocolo pós-exposição ocupacional de acidentes entre cirurgiões dentistas. Revista Saúde Pública, 2010; 44(3):529-40.

28. Marziale MHP, Nishimura KYN, Ferreira MM. Riscos de contaminação ocasionados por acidentes de trabalho com material perfuro-cortante entre trabalhadores de enfermagem. Rev. Latinoam.Enferm. 2004; 12(1): 36-42.

29. Monteiro CM, Benatti MCC, Rodrigues RCM. Acidente do trabalho e qualidade de vida relacionada à saúde: um estudo em três hospitais. Rev Latino-Am Enfermagem. 2009, 17(1).

30. Murofuse NT, Alves DCl, Fávero GC, Brotto AO. Comportamento dos acadêmicos, docentes e técnicosadministrativos da clínica odontológica da Unioeste: riscos ocupacionais e atividades desenvolvidas. Acta Sci. Health Sci. Maringá. 2008; 30(1): 81-7.

31. Murofuse NT, Marziale MHP, Gemelli LMG. Acidente com material biológico em hospital universitário do oeste do Paraná. Rev Gaúcha Enferm. 2005; 26(2): 168-79.

32. Nishide VM, Benatti MCC, Alexandre NMC. Ocorrência de acidente do trabalho em uma unidade de terapia intensiva. Rev. Latinoam. Enferm. 2004; 12(2): 204-11 
33. Oliveira AC, Gonçalves JA. Acidente ocupacional por material perfurocortante entre profissionais de saúde de um Centro Cirúrgico. Rev Esc Enferm USP 2010; 44(2): 482-7.

34. Oliveira AC, Lopes ACS, Paiva MHRS. Acidentes ocupacionais por exposição a material biológico entre a equipe multiprofissional do atendimento pré-hospitalar. Rev. Esc. Enferm. USP, 2009; 43(3): 677-83.

35. Oliveira BAC, Kluthcovsky ACGC, Kluthcovsky FA. Estudo sobre ocorrências de acidentes de trabalho com material biológico em profissionais de enfermagem de um hospital. Cogitare Enferm. 2008; 13(2): 194205.

36. Oliveira NLB, Sousa RMC. Retorno à atividade produtiva de motociclistas vítimas de acidentes de trânsito. Acta paul. enferm. 2006; 19(3): 284-9.

37. Osorio C. ACAT: o trabalhador como protagonista da análise de acidentes de trabalho. Cadernos de Psicologia Social do Trabalho. 2008; 11(1): 111-20.

38. Osorio C, Machado JMH, Minayo-Gomez C. Proposicão de um método de análise coletiva dos acidentes de trabalho no hospital. Cad. Saúde Pública. 2005; 21(2): 517-24.

39. Paulino DCR, Lopes MVO, Rolim ILTP. Biossegurança e acidentes de trabalho com perfuro-cortantes entre os profissionais de enfermagem de hospital universitário de Fortaleza-CE. Cogitare Enferm. 2008; 13(4): 507-13.

40. Pereira ACM, Silva AR, Rocha CF, Cordeiro IS, Lopes CM. . Acidentes de trabalho com material perfurocortante em profissionais da equipe de enfermagem da rede hospitalar pública de Rio Branco - Acre Brasil. Online Braz. J. Nnurs. (Online). 2004; 3(3).

41. Prado-Palos MA, Canini SRMS, Gir E, Melo LL, Mata DH, Santana RMT et al. Acidentes com Material Biológico Ocorridos com Profissionais de Laboratórios de Análises Clínicas. DST j. bras. doenças sex. transm. 2006; 18(4):231-4.

42. Ribeiro PC, Ribeiro ACC, Lima Júnior FPB. Perfil dos acidentes de trabalho em um hospital de Teresina, PI. Cogitare Enferm. 2010; 15(1): 110-6.

43. Rosin-Pinola AR, Silva CP, Garbulho NF. Implicações psicossociais para o acidentado de trabalho reinserido no mercado de trabalho e desempregado. Rev. Bras. Orientac. Prof. 2004; 5(2): 53-62.

44. Ruiz MT, Barboza DB, Soler ZASG. Acidentes de trabalho: um estudo sobre esta ocorrência em um hospital geral. Arq. Ciênc. Saúde. 2004; 11(4): 219-24.

45. Sailer GC, Marziale MHP. Vivência dos trabalhadores de enfermagem frente ao uso dos antiretroviarais após exposição ocupacional a material. Texto e Contexto Enferm. 2007; 16(1): 55-62.

46. Sarquis LMM, Felli VEA, Miranda FMA, Guimarães HV, Oliveira GP. A adesão ao protocolo de monitoramento dos trabalhadores de saúde após exposição a fluidos biológicos: uma problemática vivenciada em um ambulatório de saúde do trabalhador no Paraná. Cogitare Enferm. 2005; 10(2): 47-53.

47. Sarquis LMM, Felli VEA. Os sentimentos vivenciados após exposição ocupacional entre trabalhadores de saúde: fulcro para repensar o trabalho em instituições de saúde. Rev. Bras. Enferm. 2009; 62(5): 701-4.

48. Silveira CA, Robazzi MLCC, Walter EV, Marziale MHP. Acidentes de trabalho na construção civil identificados através de prontuários hospitalares. REM: R. Esc. Minas. 2005, 58(1): 39-44.

Saúde (Santa Maria), v.37, n.1, p. 77-90,

2011. Prochnow, A. et al.

49. Silveira CA, Robazzi MLCC, Marziale MHP, Dalri MCB. Acidente de trabalho entre trabalhadores rurais e da agropecuária identificados através de registros hospitalares. Ciência, Cuidado e Saúde. 2005; 4(2): 120- 
50. Simão SAF, Souza V, Borges RAA, Soares CRG, Cortez EA. Fatores associados aos acidentes biológicos entre profissionais de enfermagem. Cogitare Enferm. 2010; 15(1):87-91.

51. Soerensen AA, Moriya TM, Soerensen R, Robazzi MLCC. Atendimento pré-hospitalar móvel: fatores de riscos ocupacionais. Rev. Enferm. UERJ. 2008, 16(2): 187-92.

52. Teixeira CS, Pasternak-Júnior B, Silva- Sousa YTC, Correa-Silva SR. Medidas de prevenção pré e pósexposição a acidentes perfurocortantes na prática odontológica. Rev. odonto ciênc. 2008; 23(1): 10-4.

53. Zangirolani LTO, Cordeiro R, Medeiros MAT, Stephan C. Topologia do risco de acidentes do trabalho em Piracicaba, SP. Rev. Saúde Pública. 2008; 42(2): 287-93. 